\title{
Ventilator-associated pneumonia due to colistin susceptible-only microorganisms
}

\author{
F.G. Rios*, C.M. Luna\#, B. Maskin*, A. Saenz Valiente*, M. Lloria", S. Gando\#, \\ C. Sosa ${ }^{\#}$, S. Baquero ${ }^{\#}$, C. Llerena ${ }^{*}$, C. Petrati ${ }^{\star}$ and C. Apezteguia*
}

ABSTRACT: Acinetobacter spp. and Pseudomonas aeruginosa are common pathogens of ventilator-associated pneumonia (VAP). The presentation and outcome of VAP due to Acinetobacter spp. and $P$. aeruginosa susceptible to carbapenems (Carb-S; imipenem and/or meropenem) and to colistin only (Col-S) were compared in the present retrospective study in three intensive care units.

A total of 61 episodes of VAP caused by Acinetobacter spp. or $\boldsymbol{P}$. aeruginosa were studied, of which 30 isolates were Carb-S and 31 were Col-S.

Demographics, worsening of renal function and mortality were not different. The univariate analysis showed that a later onset and a previous episode of VAP, prior antimicrobial therapy for $>10$ days and previous therapy with carbapenems during the present admission were more frequent in patients with Col-S strains. On multivariate analysis, prior antimicrobial therapy for $>10$ days and a previous episode of VAP remained significantly associated with Col-S VAP. Approximately $41 \%$ of the infections caused by Col-S isolates, but none of those due to Carb-S isolates, had received prior carbapenem therapy.

Colistin-susceptible ventilator-associated pneumonia episodes can be effectively treated using colistin without significant renal dysfunction. This susceptibility pattern could be suspected in patients with a previous ventilator-associated pneumonia episode or prior antibiotic therapy for $>10$ days preceding the present ventilator-associated pneumonia episode.

KEYWORDS: Antibiotic resistance, antibiotic treatment of pneumonia, bacterial infections, ventilator-associated pneumonia

$\mathrm{T}$ he isolation of multiple drug resistant (MDR) Acinetobacter spp. and Pseudomonas aeruginosa is an increasing phenomenon observed in different intensive care units (ICUs) around the world [1-3]. In recent years, strains of Acinetobacter spp. and $P$. aeruginosa nonsusceptible to nearly all classes of drugs, including carbapenems, one of the most effective antimicrobials for these pathogens [3, 4], were identified more frequently as the pathogens of ventilator-associated pneumonia (VAP) [2, 3, 5]. Colistin appears as an appropriate therapeutic alternative. The amount of data available regarding epidemiological and clinical characteristics of VAP due to Gram-negative bacilli susceptible only to colistin (Col-S) is limited [6, 7]. The mortality rate of VAP increases when the initial antimicrobial therapy is inappropriate [8-11], so it is extremely important to identify patients at risk and to begin the appropriate empiric antimicrobial therapy as soon as possible.

Colistin is an antibiotic of the polymyxin family and it is produced by Bacillus colistinus. It has been available since 1959 for the treatment of infections caused by Gram-negative bacteria [12] It is thought to act by altering the cell wall permeability producing bacterial lysis [13]. In vitro colistin has a broad spectrum of action against Gram-negative bacteria, including some strains resistant to penicillins, carbapenems, aminoglycosides and fluoroquinolones. However, Proteus mirabilis, Providencia spp., Serratia spp., Burkholderia cepacia and Stenotrophomonas maltophilia are naturally nonsusceptible to colistin [14]. Severe adverse effects (i.e. nephrotoxicity and neurotoxicity) have been reported which led to the discontinuation of parenteral use of this drug in the 1970s [7, 14]. Acquisition of resistance to colistin is uncommon; it has been described in cystic fibrosis patients chronically treated with nebulised colistin for tracheal colonisation with MDR $P$. aeruginosa. Sodium colistin methanesulphonate is the commercially available form for i.v. use [15].

Since colistin has been associated with severe adverse effects and an inappropriate initial

\section{AFFILIATIONS}

${ }^{*}$ Critical Care Service, Profesor Alejandro Posadas Hospital, Haedo, "Pulmonary and Critical Care Divisions, Hospital de Clínicas, Buenos Aires University, and "Critical Care Service, HIGA Eva Perón Hospital, San Martin, Buenos Aires, Argentina.

CORRESPONDENCE

C.M. Luna

Acevedo 1070

Banfield (1828)

Buenos Aires

Argentina

Fax: 541142423066

E-mail: cymluna@fmed.uba.ar

Received:

December 032006

Accepted after revision:

April 222007

STATEMENT OF INTEREST

None declared.

European Respiratory Journa Print ISSN 0903-1936 Online ISSN 1399-3003 
empirical antibiotic treatment is consistently associated with increased mortality, the identification of factors associated to Acinetobacter spp. and $P$. aeruginosa Col-S, compared with strains susceptible to other antibiotics, would assist in choosing the initial antimicrobial therapy.

The aim of the present study was to compare the epidemiological conditions and clinical presentation of patients with VAP caused by MDR Acinetobacter spp. or P. aeruginosa that preserve their susceptibility to carbapenems (Carb-S) with Col$\mathrm{S}$ strains, focusing on worsening serum creatinine concentration identified during therapy.

\section{METHODS}

The present retrospective study was based on the clinical and microbiological records of ICU patients from three different hospitals (Profesor Alejandro Posadas Hospital, Hospital de Clínicas and HIGA Eva Perón Hospital) located in the metropolitan area of Buenos Aires, Argentina, during a 26month period (December 2001-January 2004). Episodes of VAP caused by Carb-S or Col-S Acinetobacter spp. or P. aeruginosa, fulfilling the inclusion criteria detailed hereafter, were studied. In these ICUs, the rate of VAP due to MDR microorganisms was $\sim 69 \%$, according to a previous multicentre prospective study on VAP [16]. In such previous studies, VAP incidence in mechanically ventilated patients was $14.8 \%$, of which $\sim 40 \%$ was due to Acinetobacter spp. or P. aeruginosa and one third was due to Col-S. Empirical antimicrobial therapy included a carbapenem in $\sim 60 \%$ of the cases and other $\beta$-lactams were used in most of the remaining cases. In the present study, a combined therapy with an aminoglycoside, a fluoroquinolone in combination with a carbapenem, or colistin was used in 12 cases as empiric therapy. In 16 cases, colistin was used after the culture report showing Col-S Acinetobacter spp. or $P$. aeruginosa. In the remaining 15 cases, the initial therapy with colisitin was empirical according to the decision of the attending physician.

Inclusion criteria were as follows. 1) Aged $>18$ yrs with a new or progressive pre-existent radiographic infiltrate. 2) The isolation, bronchoscopically obtained, of $\geqslant 10^{4}$ colony forming units $(\mathrm{cfu}) \cdot \mathrm{mL}^{-1}$ of MDR Acinetobacter spp. or P. aeruginosa from bronchoalveolar lavage (BAL) fluid or $10^{3} \mathrm{cfu} \cdot \mathrm{mL}^{-1}$ of MDR Acinetobacter spp. or $P$. aeruginosa from protected specimen brush (PSB). 3) Presenting with at least one of the following: purulent secretions, abnormal temperature $\left(>38^{\circ} \mathrm{C}\right.$ or $\left.<36^{\circ} \mathrm{C}\right)$ and/or abnormal leukocyte count $(>10,000$ or $<4,000$ white blood cell count $\cdot \mathrm{mm}^{-3}$ ). Microorganisms were considered MDRs when they were resistant to two or more antimicrobial classes to which they are naturally susceptible. The Carb-S Acinetobacter spp. or P. aeruginosa group includes VAPs due to MDR organisms that were consistently susceptible to imipenem and/or meropenem. The Col-S Acinetobacter spp. or $P$. aeruginosa group includes VAPs due to MDR organisms that were susceptible to colistin but resistant to carbapenems and to all other parenteral available antibiotics.

All patients initiated antimicrobial therapy empirically. Treatment was eventually modified when pathogens were identified and their antimicrobial susceptibilities were available (definitive therapy). The onset of appropriate antibiotic therapy, empiric or definitive, was taken into account to evaluate the therapy and to determine its duration and the time elapsed until the detection of adverse events. The definitive therapy for patients with VAP due to Carb-S bacteria consisted of imipenem $2 \mathrm{~g} \cdot \mathrm{day}^{-1}(\mathrm{n}=11)$ or meropenem $3 \mathrm{~g} \cdot \mathrm{day}^{-1}(\mathrm{n}=19)$, while patients with VAP due to Col-S were treated with i.v. colistin $5 \mathrm{mg} \cdot \mathrm{kg}^{-1} \cdot \mathrm{day}^{-1}$. Doses were corrected in patients with renal failure according to formulas published elsewhere [7].

Demographic data, Acute Physiology and Chronic Health Evaluation (APACHE) II score at ICU admission, underlying diseases, aetiological agent, reasons for mechanical ventilation, previous VAP episodes and number of days spent in hospital, in the ICU and on mechanical ventilation (before and after the diagnosis of VAP), were recorded. Data on previous antimicrobial therapy during the 10 days preceding VAP onset, reasons for such antimicrobial therapy, days receiving antibiotics for VAP with carbapenems or colistin and the impact on renal function were also taken into consideration. Since the initiation of colistin or carbapenem could happen 2-3 days after the diagnosis of VAP, when the result of cultures and antimicrobial susceptibility studies became available, serum creatinine change during follow-up was evaluated taking into account the day of onset of appropriate antibiotic therapy.

Serum creatinine concentration was obtained at the onset of appropriate therapy and 3, 5, 7, 10 and 14 days later. The bacterial antimicrobial susceptibility was established by disk diffusion. It was considered that antimicrobial therapy was inappropriate when the isolated microorganisms were not susceptible to the initial empiric antimicrobial therapy. A 28 day overall mortality after the diagnosis of VAP was evaluated.

\section{Statistical analysis}

Data are noted in absolute numbers, with or without percentages, as mean \pm SD or as medians with 1-3 quartiles. Unpaired t-tests were used to compare continuous variables, whereas Chi-squared or Fischer exact tests were used to compare categorical data and proportions. Univariate and multivariate analysis with forward stepwise logistic regression analysis for the variables that were significantly different in patients with Col-S, were applied in order to better understand the risk factors related to the development of Col-S VAP episodes. Variables were entered into the model when $\mathrm{p}<0.05$. Adjusted odds ratios and $95 \%$ confidence intervals (CI) were calculated. The variation of serum creatinine concentration at $3,5,7,10$ and 14 days compared with the day of onset of antimicrobial therapy was recorded in patients receiving colistin or carbapenems by using a two-way ANOVA.

\section{RESULTS}

A total of 61 patients fulfilling the clinical and microbiological criteria for MDR Acinetobacter spp. or P. aeruginosa VAP in the BAL $(n=55)$ or in the PSB culture $(n=6)$ were included. Of the 61 cases, 30 were due to Carb-S and 31 to Col-S strains. Demographic and clinical data of the two groups are displayed in table 1. More than one pathogen was isolated in 11 specimens (1.18 per patient; table 2). Among the remaining 50 cases, 36 were due to Acinetobacter spp. and 14 were due to $P$. aeruginosa. All the Carb-S pathogens were susceptible to 


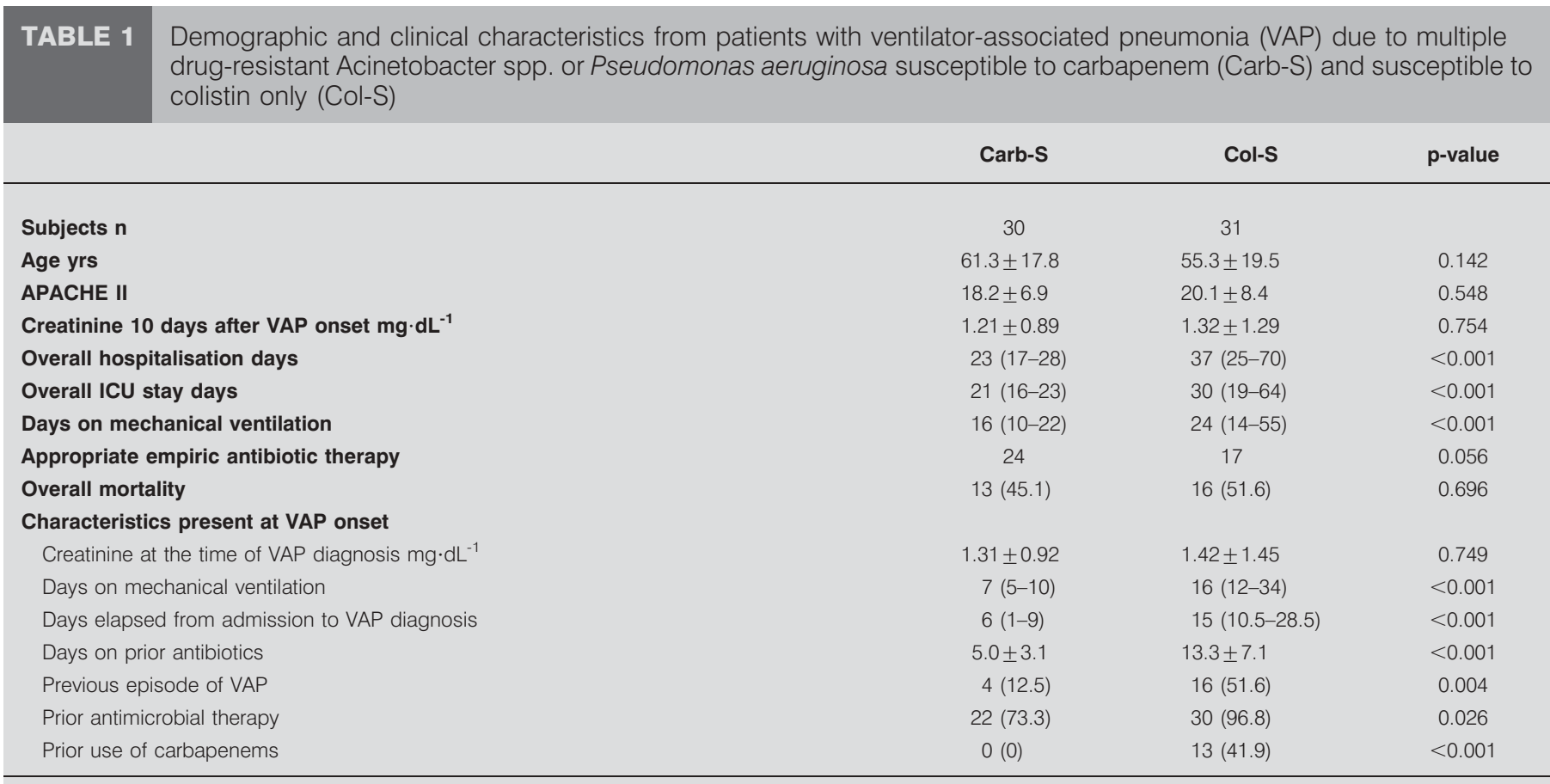

Data are presented as mean \pm SD, mean (range) or $n(\%)$, unless otherwise stated. APACHE: Acute Physiology and Chronic Health Evaluation; ICU: intensive care unit.

imipenem and/or meropenem and to colistin. Additionally, among the 20 Carb-S Acinetobacter spp. isolates, nine were susceptible to ampcillin-sulbactam, three to piperacillin and/ or tazobactam, two to levofloxacin and one to amikacin. Among the 11 Carb-S P. aeruginosa isolates (two cases with mixed aetiology due to Acinetobacter spp. and P. aeruginosa), six were susceptible to piperacillin and/or tazobactam, four to cefoperazone, three to ciprofloxacin, three to amikacin, two to levofloxacin and two to gentamicin. The 26 Acinetobacter spp. isolates and $11 \mathrm{P}$. aeruginosa Col-S isolates (seven cases with mixed aetiology due to Acinetobacter spp. and $P$. aeruginosa) were resistant to all the other parenteral available antibiotics (a small number were susceptible to minocycline, not available for parenteral use). In total, 29 patients died within 28 days

\begin{tabular}{lccc} 
TABLE 2 & $\begin{array}{l}\text { Microorganisms isolated in respiratory } \\
\text { specimens obtained by bronchoalvelolar } \\
\text { lavage }{ }^{\#} \text { or protected specimen brush }\end{array}$ & \\
& Total & Carb-S & Col-S \\
\hline Subjects & 61 & 30 & 31 \\
Acinetobacter baumannii & 46 & 20 & 26 \\
Pseudomonas aeruginosa & 24 & 13 & 11 \\
Staphylococcus aureus & 1 & 1 & \\
Klebsiella pneumoniae & 1 & 1 & \\
Microorganisms per VAP episode & 1.18 & 1.17 & 1.19 \\
\hline
\end{tabular}

Data are presented as n. Carb-S: carbapenems susceptible; Col-S: colistin susceptible only; VAP: ventilator-associated pneumonia. ${ }^{\#}: n=55 ;{ }^{\natural}: n=6$. after VAP onset (mortality $=47.5 \%$ ). Mortality rates for Acinetobacter spp. and P. aeruginosa were 41.7 and $61.3 \%$, respectively $(p=0.361)$, when they were the only isolated microorganism. Length of hospital stay after VAP diagnosis in survivors was $45.8 \pm 110.2$ days. Admissions were due to medical $(62 \%)$ and surgical $(38 \%)$ reasons. Demographic and clinical data from the patients for the Carb-S and Col-S groups of patients are displayed in table 2 .

\section{Antimicrobial therapy}

The duration of therapy in patients who survived $\geqslant 15$ days was $12.0 \pm 6.2$ and 12.2 \pm 5.8 days for the Carb-S group and the Col-S group, respectively. No difference was observed in the mortality rate between the Carb-S and the Col-S groups, but hospital and ICU length of stay were significantly longer for patients in the Col-S group (table 1). In most cases, initial empirical antimicrobial therapy included a $\beta$-lactam active against MDR Gram-negative bacilli (carbapenem or piperacillin-tazobactam). Some patients also received an aminoglycoside or ciprofloxacin and/or colistin as part of this empirical antimicrobial therapy. In 20 patients this therapy was inappropriate, 14 from the Col-S group and six from the Carb-S group ( $p=$ nonsignificant). However, a significantly higher mortality rate was identified in patients who received an initial inappropriate therapy (70 versus $36.6 \%$; $p=0.014$ ). This difference persisted in patients who had switched to an appropriate therapy a few days after the starting point (fig. 1). The mortality rate for the VAP episodes caused by Acinetobacter spp. or $P$. aeruginosa treated with appropriate empiric antimicrobial therapy was exactly the same (i.e. $33.3 \%$; eight out of 24 patients and three out of nine patients, respectively). 


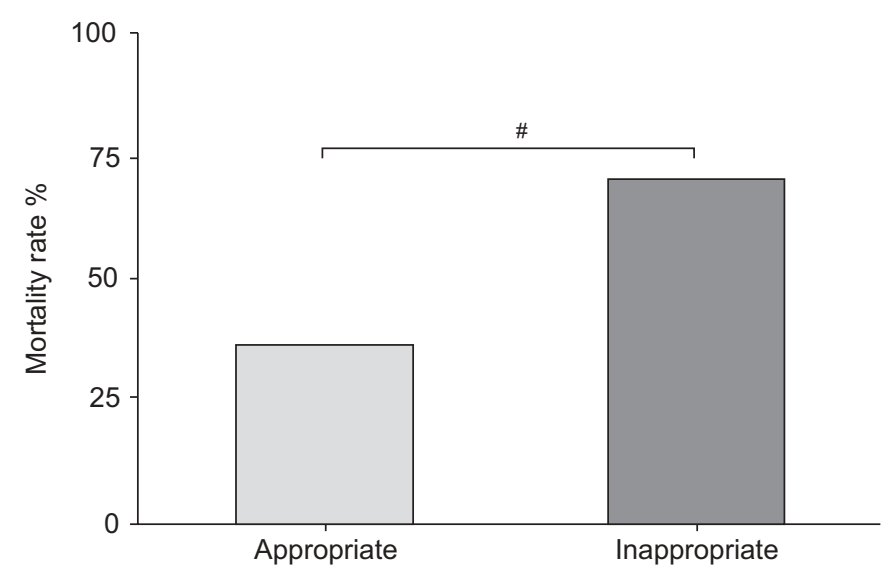

FIGURE 1. Mortality rates in the overall population of 61 patients related to empirically prescribed antimicrobial therapy appropriateness $(36.6 \%$ in the appropriate therapy and $70.0 \%$ in the inappropriate therapy groups, respectively) at the time of ventilator-associated pneumonia diagnosis. The therapeutic measure taken prior to confirming the nature of the microorganisms involved and their antimicrobial susceptibility was taken into account. Appropriate antimicrobial therapy was administered more commonly to patients with carbapenemssusceptible rather than colistin-susceptible pneumonia. ${ }^{*}: p=0.014$.

\section{Other characteristics during hospitalisation}

The APACHE II score at ICU admission and the mortality rate were similar in both groups (table 1). In the present cohort, hospitalisation was very long (28 (23-41) days). During the ICU stay (23 (17-34) days), patients remained on mechanical ventilation for 19 (14-28) days. Comparing both VAP groups, hospitalisation time, time in ICU, days on mechanical ventilation and days in the ICU before VAP onset were significantly shorter in patients with Carb-S isolate compared with those Col-S Acinetobacter spp. or P. aeruginosa (table 1). Prior antimicrobial treatment, particularly with carbapenems, and a previous VAP history were significantly more frequent in the Col-S groups.

\begin{tabular}{|c|c|c|c|c|}
\hline \multirow[t]{2}{*}{ TABLE 3} & \multicolumn{4}{|c|}{$\begin{array}{l}\text { Reasons for prior antimicrobial therapy in } 52 \\
\text { patients }\end{array}$} \\
\hline & & Carb-S & Col-S & p-value \\
\hline \multicolumn{2}{|l|}{ Subjects } & 21 & 31 & \\
\hline \multicolumn{2}{|c|}{ Nosocomial pneumonia including VAP } & 1 & 15 & 0.001 \\
\hline \multicolumn{2}{|c|}{ Community-acquired pneumonia } & 5 & 7 & 1.000 \\
\hline \multicolumn{2}{|c|}{ Intra-abdominal infection } & 3 & 5 & 1.000 \\
\hline \multicolumn{2}{|c|}{ Other lower respiratory tract infections } & 4 & 1 & 0.145 \\
\hline \multicolumn{2}{|c|}{ Acute exacerbation of COPD } & 4 & 0 & 0.022 \\
\hline \multicolumn{2}{|c|}{ Bacteremia or sepsis } & 1 & 2 & 1.000 \\
\hline \multicolumn{2}{|c|}{ Miscellaneous } & 3 & 1 & 0.291 \\
\hline
\end{tabular}

Data are presented as n. Carb-S: carbapenems susceptible; Col-S: colistin susceptible; VAP: ventilator-associated pneumonia; COPD: chronic obstructive pulmonary disease.

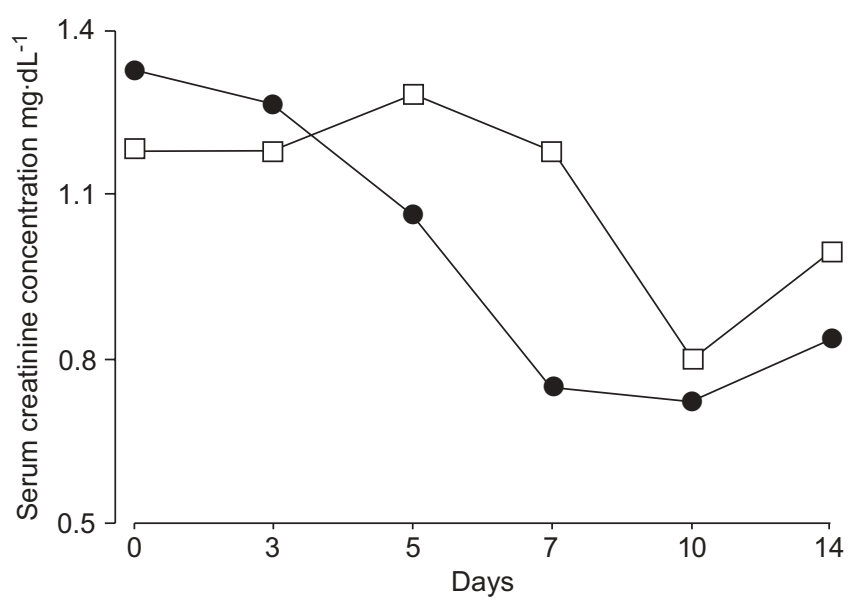

FIGURE 2. Mean serum creatinine concentration evolution in both carbapenem-susceptible $(\square)$ and colistin-susceptible (-) groups from the onset of ventilator-associated pneumonia to 14 days after administration of the initial appropriate antimicrobial therapy (carbapenem or colistin). There was no difference in the serum creatinine concentration between the two groups compared (two-way ANOVA).

In total, 30 Col-S and 25 Carb-S patients had received antimicrobial therapy prior to VAP diagnosis $(\mathrm{p}=0.012)$. The duration of prior therapy was $9.2 \pm 6.8$ days $(5.2 \pm 2.5$ and $12.3 \pm 4.3$ days for the Carb-S and the Col-S groups, respectively; $\mathrm{p}<0.0001)$. The two most common causes for prior antimicrobial treatment were community- and hospitalacquired respiratory tract infections. All antimicrobial therapy causes are listed in table 3. Approximately $41 \%$ of the Col-S episodes, but none of the Carb-S episodes, had received prior carbapenem therapy.

\section{Renal function evaluation}

By the time of onset of appropriate VAP therapy, the mean serum creatinine concentration was within the normal range $\left(1.32 \pm 1.19 \mathrm{mg} \cdot \mathrm{dL}^{-1}\right)$ and there was no difference between the Carb-S and Col-S groups. Comparing the value observed at onset of appropriate therapy with the levels observed 3, 5, 7 and 10 days later, there was a trend towards a reduction of serum creatinine concentration in both groups (fig. 2). Twelve (seven in the Carb-S and five in the Col-S group) patients had an initial serum creatinine level $>1.4 \mathrm{mg} \cdot \mathrm{dL}^{-1}$ (normal limit). On days 3 and 5, there were five (two Carb-S and three Col-S) and six (two Carb-S and four Col-S) patients, respectively, with a creatinine level $\geqslant 1.4 \mathrm{mg} \cdot \mathrm{dL}^{-1}$. Considering the 34 patients with normal renal function at onset of appropriate antibiotic therapy remaining in the study for $\geqslant 5$ days, and taking into account the creatinine level on days 0,3 and 5, it was observed that on day 3 , there were four patients with a creatinine level $\geqslant 1.4 \mathrm{mg} \cdot \mathrm{dL}^{-1}$ (two in each group) and on day 5 there were two patients in each group with a creatinine level $\geqslant 1.4 \mathrm{mg} \cdot \mathrm{dL}^{-1}$. Four patients (one in the Carb-S and three in the Col-S group) had a serum creatinine value $\geqslant 4.0 \mathrm{mg} \cdot \mathrm{dL}^{-1}$, but none of them presented worsened renal function during the antimicrobial therapy (data not shown). None of the 61 patients who participated in the present study required dialysis either at onset or during the follow-up of VAP. 


\begin{tabular}{|c|c|c|c|c|c|}
\hline \multirow[t]{3}{*}{ TABLE 4} & \multicolumn{5}{|c|}{$\begin{array}{l}\text { Categorical variables significantly associated with colistin-susceptible ventilator-associated pneumonia (VAP) in } \\
\text { univariate and multivariate analysis }\end{array}$} \\
\hline & & \multicolumn{2}{|c|}{ Univariate analysis } & \multicolumn{2}{|c|}{ Multivariate analysis } \\
\hline & & OR $(95 \% \mathrm{Cl})$ & p-value & $\beta$-coefficient $(95 \% \mathrm{Cl})$ & p-value \\
\hline \multicolumn{2}{|c|}{ Duration of mechanical ventilation $>\mathbf{3 0}$ days } & $17.7(1.7-179.4)$ & 0.016 & & \\
\hline \multicolumn{2}{|c|}{ Overall ICU stay $>40$ days } & $20.5(2.0-205.5)$ & $<0.001$ & $31.6(31.5-495.9)$ & 0.014 \\
\hline \multicolumn{6}{|c|}{ Characteristics present at VAP onset } \\
\hline \multicolumn{2}{|c|}{ Duration of prior antimicrobial therapy $>10$ days } & $31.5(2.9-340.4)$ & $<0.001$ & $13.2(2.2-78.7)$ & 0.005 \\
\hline \multicolumn{2}{|c|}{ Time from admission to VAP diagnosis $>16$ days } & $27.1(2.7-269.5)$ & $<0.001$ & & \\
\hline \multicolumn{2}{|c|}{ Previous episode of VAP } & $27.7(2.8-273.5)$ & $<0.001$ & $6.0(1.0-35.7)$ & 0.047 \\
\hline \multicolumn{2}{|c|}{ Prior use of carbapenems } & $21.7(2.2-212.9)$ & $<0.001$ & & \\
\hline
\end{tabular}

OR: odds ratio; $\mathrm{Cl}$ : confidence interval; ICU: intensive care unit.

\section{Multivariate analysis}

A multivariate logistic regression model was applied to the categorical variables to disclose which conditions were significantly related to Col-S isolates. The forward stepwise model using the therapy with colistin or carbapenems as the dependent variable demonstrated that duration of prior antimicrobial therapy $>10$ days remained significantly associated with the Col-S condition at VAP onset. Overall ICU stay for $>40$ days was also associated to Col-S VAP (table 4).

\section{DISCUSSION}

Although i.v. colistin is accepted as an antimicrobial option for specific MDR pathogens [17], the clinical experience with this antibiotic is poor. In fact, it has practically been discontinued due to the occurrence of serious adverse effects and the availability of other suitable alternatives. The development of MDR Col-S Gram-negative infections, particularly in critically ill patients, has led to a renewed interest on this drug.

It has been acknowledged that VAP episodes caused by the socalled "high-risk pathogens", particularly the nonfermenting Gram-negative bacilli Acinetobacter spp. and P. aeruginosa, are associated with a higher mortality rate when compared with other microorganisms. A relative risk of 2.5 has been recorded [18]. The increased incidence of nosocomial infections due to MDR pathogens has grown impressively during recent decades [19-23]. This microbiological data has resulted in a more frequent use of inappropriate antimicrobial therapy $[8,10,24]$. Imipenem and meropenem have been the most effective antimicrobials against Acinetobacter spp. and $P$. aeruginosa during the last decade. Resistance to these antimicrobials is nearly always associated with nonsusceptibility to other $\beta$-lactams, fluoroquinolones and aminoglycosides $[3,4]$. It remains unknown whether bacterial resistance to carbapenems has an intrinsic higher risk of morbidity or mortality. Similarly to previous studies, the results of the present study concerning this topic indicate that the mortality rate is comparable between patients with severe infections due to Carb-S and Col-S Acinetobacter spp. or P. aeruginosa [6, 25]. It was observed, however, that inappropriate therapy was consistently associated with higher mortality, regardless of which antimicrobial was used.
TROUILLET et al. [21] identified several factors related to the acquisition of VAP due to MDR microorganisms, including a duration of mechanical ventilation $>7$ days, prior antimicrobial therapy and the use of broad-spectrum antibiotics. Similarly, Rios et al. [22] identified hospitalisation prior to $\mathrm{VAP}$ onset during $>5$ days and the use of prior antimicrobial therapy as risk predictors of VAP caused by MDR pathogens.

Reports about colistin-related toxicity pointed out a variable incidence and established that their adverse effects are transient [6, 26, 27]. CONWAY et al. [28] considered that the first reports of adverse events could be erroneously attributed to colistin due to the complexity of the clinical background observed in those patients. Aminoglycosides and glycopeptidic antimicrobials are commonly used in critically ill patients, but these highly effective antibiotics may also produce nephrotoxicity in a large number of patients [29, 30]. The knowledge of the pharmacokinetics and pharmacodynamics of colistin administered intravenously is limited. In an experimental mice comparative model about the efficacy of different antibiotics against pneumonia caused by MDR Acinetobacter spp., MONTERO et al. [31] reported that, disregarding its in vitro microbiological activity according to its minimum inhibitory concentration, the results were discouraging with regards to the use of colistin to treat Acinetobacter spp. pneumonia. These results require careful interpretation and any extrapolation to humans should be done with caution.

Several studies on efficacy and/or safety of parenteral colistin for critically ill patients with VAP or other severe infections due to MDR Acinetobacter spp. or $P$. aeruginosa have been published in recent years [6, 25, 32, 33]. GARNACHO-MONTERO et al. [6] compared 21 carbapenem-resistant strains of Acinetobacter spp. VAP episodes treated with colistin with 14 carbapenem-susceptible VAP episodes treated with imipenem, but no differences were found regarding efficacy, nephrotoxicity or neurotoxicity. LINDEN et al. [33] studied 23 critically ill patients infected with MDR P. aeruginosa and treated with colistin, including 18 who had pneumonia. Out of these 23 patients, 14 had a favourable clinical response. In that study, bacteremia was the only factor associated with therapeutic failure. KASIAKOU et al. [32] studied 54 episodes 
of severe infections in cystic fibrosis patients due to MDR Gram-negative bacteria treated with a combination therapy, which included parenteral colistin. It was reported that $8 \%$ of the patients worsened their renal function during therapy, while $67 \%$ improved or were cured, showing colistin's safety and effectiveness. Finally, in a study of 185 critically ill patients with Acinetobacter spp. or $P$. aeruginosa infections (55 were treated with colistin and 105 of the remaining 130 were treated with a carbapenem), REINA et al. [25] concluded that colistin was as safe and effective as other antibiotics. However, the authors did not find an association between inappropriate antimicrobial therapy, more common in the colistin group, and poorer outcome.

Regarding renal function, serum creatinine concentration was thoroughly examined. No significant adverse effects attributable to colistin were found in the present cohort. Regarding patients with normal renal function at the onset of appropriate antibiotic therapy, a few of them showed a mild and transient elevation of serum creatinine levels. Approximately half of the patients that presented an abnormal initial serum creatinine concentration observed a decrease in their levels during colistin therapy.

Multivariate analysis demonstrated that previous VAP episodes and prior antimicrobial therapy for $>10$ days are risk factors for VAP due to Col-S P. aeruginosa or Acinetobacter spp. This group also showed a longer stay in the ICU.

Delay in the initiation of antimicrobial therapy increases the VAP mortality rate $[29,34]$. A higher mortality rate was found in patients who did not receive appropriate therapy or received it with delay, in comparison with those that received appropriate therapy. It is believed that in patients with a high risk of harbouring MDR nonfermenting Gram-negative bacteria admitted to ICUs with similar epidemiological conditions to patients included in the present study, it could be appropriate to begin the empiric initial antimicrobial therapy using colistin. In fact, the current American Thoracic Society/ Infectious Diseases Society of America guidelines for hospitalacquired, ventilator-associated and healthcare-associated pneumonia recommend considering colistin as a therapy for patients with VAP attributed to carbapenem-resistant Acinetobacter spp. [17].

The present study has several limitations. It is a retrospective study on a relatively reduced number of patients that were recruited at three different institutions, thus many factors were not controlled and it may present biases inherent to this kind of study. The analysis of Acinetobacter spp. and P. aeruginosa together could be inappropriate, as these are different microorganisms and could have different response to therapy. A prospective randomised study with more patients is necessary to analyse in depth the factors associated with the development of Col-S Acinetobacter spp. or P. aeruginosa VAP and to confirm the findings of the present study. The results of the current study are limited by the high incidence of MDR pathogens in the participating institutions and the local ICU practice, as evidenced by a high rate of carbapenem use as empiric therapy for VAP (24 Carb-S patients received carbapenems empirically, while 12 Col-S patients had also received previous carbapenem therapy). Carbapenems often represent the last line of $\beta$-lactam therapy due to their greater resilience against common resistance mechanisms (e.g. $\beta$ lactamases).

Colistin should not always be recommended as first-line therapy. Physicians should know their own ecology and should only consider colistin use as an option when there are risk factors for Col-S isolates and, as a consequence, there is no other choice (i.e. highly selected patients with a previous VAP episode and prior use of antimicrobial therapy, especially carbapenems, for $>10$ days).

In summary, patients with multiple drug-resistant colistinsusceptible Pseudomonas aeruginosa or Acinetobacter spp. ventilator-associated pneumonia are characterised by presenting some of the following: previous ventilator-associated pneumonia episodes; prior broad-spectrum antimicrobial therapy for $>10$ days (particularly carbapenems); and delayed onset of ventilator-associated pneumonia from the intensive care unit admission. Under these conditions, in intensive care units with the ecological characteristics described in the present study, colistin could be a suitable antibiotic for initial empiric antimicrobial therapy.

\section{REFERENCES}

1 Crouch Brewer S, Wunderink RG, Jones CB, Leeper KV Jr. Ventilator-associated pneumonia due to Pseudomonas aeruginosa. Chest 1996; 109: 1019-1029.

2 Rahal JJ, Urban C. Acinetobacter. Semin Respir Crit Care Med 2000; 21: 341-348.

3 Gales AC, Jones RN, Forward KR, Linares J, Sader HS, Verhoef J. Emerging importance of multi-drug resistant Acinetobacter species and Stenotrophomonas maltophilia as pathogens in seriously ill patients: geographic patterns, epidemiological features, and trends in the SENTRY Antimicrobial Surveillance Program (1997-1999). Clin Infect Dis 2001; 32: Suppl. 2, S104-S113.

4 Gales AC, Jones RN, Turnidge J, Rennie R, Ramphal R. Characterization of Pseudomonas aeruginosa isolates: occurrence rates, antimicrobial susceptibility patterns, and molecular typing in the global SENTRY Antimicrobial Surveillance Program, 1997-1999. Clin Infect Dis 2001; 32: Suppl. 2, S146-S155.

5 Ríos F, Luna CM, Menga G, et al., Análisis de neumonía asociada a ventilación mecánica en 6 hospitales de Buenos Aires. [Analysis of ventilator-associated pneumonia in 6 hospitals of Buenos Aires.] 13th Argentinian Congress of Intensive Therapy. September 2002, Buenos Aires, Argentina.

6 Garnacho-Montero J, Ortiz-Leyba C, Jimenez-Jimenez FJ, et al. Treatment of multidrug-resistant Acinetobacter baumannii ventilator-associated pneumonia (VAP) with intravenous colistin: a comparison with imipenem-susceptible VAP. Clin Infect Dis 2003; 36: 1111-1118.

7 Levin AS, Barone AA, Penco J, et al. Intravenous colistin as therapy for nosocomial infections caused by multidrugresistant Pseudomonas aeruginosa and Acinetobacter baumannii. Clin Infect Dis 1999; 28: 1008-1011.

8 Luna CM, Vujacich P, Niederman MS, et al. Impact of BAL data on the therapy and outcome of ventilator-associated pneumonia. Chest 1997; 111: 676-685. 
9 Celis R, Torres A, Gatell JM, Almela M, RodriguezRoisin R, Agusti-Vidal A. Nosocomial pneumonia. A multivariate analysis of risk and prognosis. Chest 1988; 93: 318-324.

10 Kollef $\mathrm{MH}$, Sherman G, Ward S, Fraser VJ. Inadequate antimicrobial treatment of infections. A risk factor for hospital mortality among critically ill patients. Chest 1999; 115: 462-474.

11 Rello J, Ausina V, Ricart M, Castella J, Prats G. Impact of previous antimicrobial therapy on the etiology and outcome of ventilator-associated pneumonia. Chest 1993; 104: 1230-1235.

12 Ross S, Puig JR, Zaremba EA. Colistin: some preliminary laboratory and clinical observations in specific gastroenteritis in infants and children. Antibiot Annu 1959- 1960; 7: 89-100.

13 Polimixina-Colistina. [Polymyxin-Colistin.] In: Bergoglio RM, ed. Antibióticos [Antibiotics.]. Buenos Aires, Editorial Médica Panamericana, 1993; pp. 254-259.

14 Catchpole CR, Andrews JM, Brenwald N, Wise R. A reassessment of the in vitro activity of colistin sulphomethate sodium. J Antimicrob Chemother 1997; 39: 255-260.

15 Li J, Nation RL, Milne RW, Turnidge JD, Coulthard K. Evaluation of colistin as an agent against multi-resistant Gram-negative bacteria. Int J Antimicrob Agents 2005; 25: 11-25.

16 Luna CM, Blanzaco D, Niederman MS, et al. Resolution of ventilator-associated pneumonia: prospective evaluation of the clinical pulmonary infection score as an early clinical predictor of outcome. Crit Care Med 2003; 31: 676-682.

17 American Thoracic Society, Infectious Diseases Society of America. Guidelines for the management of adults with hospital-acquired, ventilator-associated, and healthcareassociated pneumonia. Am J Respir Crit Care Med 2005; 171: 388-416.

18 Chastre J, Fagon JY. Pneumonia in the ventilator-dependent patient. In: Tobin MJ, ed. Principles and Practice of Mechanical Ventilation. New York, McGraw-Hill Inc., 1994; pp. 857-890.

19 Levin AS. Multiresistant Acinetobacter infections: a role for sulbactam combinations in overcoming an emerging worldwide problem. Clin Microbiol Infect 2002; 8: 144-153.

20 Chastre J, Trouillet JL, Vuagnat A, et al. Nosocomial pneumonia in patients with acute respiratory distress syndrome. Am J Respir Crit Care Med 1998; 157: 1165-1172.

21 Trouillet JL, Chastre J, Vaugnat A, et al. Ventilatorassociated pneumonia caused by potentially drug-resistant bacteria. Am J Respir Crit Care Med 1998; 157: 531-539.

22 Rios F, Luna CM, Lucini O, et al. Multiresistant pathogens and antimicrobial prescribing practices for ventilator-associated pneumonia (VAP). Impact of prior duration of mechanical ventilation (MV) or staying and antibiotics use. Chest 2002; 122: 6S-7S.

23 Craig CP, Connelly S. Effect of intensive care unit nosocomial pneumonia on duration of stay and mortality. Am J Infect Control 1984; 12: 233-238.

24 Iregui M, Ward S, Sherman G, Fraser VJ, Kollef MH. Clinical importance of delays in the initiation of appropriate antibiotic treatment for ventilator-associated pneumonia. Chest 2002; 122: 262-268.

25 Reina R, Estenssoro E, Saenz G, et al. Safety and efficacy of colistin in Acinetobacter and Pseudomonas infections: a prospective cohort study. Intensive Care Med 2005; 31: 10581065.

26 Wolinsky E, Hines JD. Neurotoxic and nephrotoxic effects of colistin in patients with renal disease. N Engl J Med 1962; 266: 759-762.

27 Koch-Weser J, Sidel VW, Federman EB, Kanarek P, Finer DC, Eaton AE. Adverse effects of sodium colistimethate. Manifestations and specific reaction rates during 317 courses of therapy. Ann Intern Med 1970; 72: 857-868.

28 Conway SP, Pond MN, Watson A, Etherington C, Robey HL, Goldman MH. Intravenous colistin sulphomethate in acute respiratory exacerbations in adult patients with cystic fibrosis. Thorax 1997; 52: 987-993.

29 Le Moyec L, Racine S, Le Toumelin P, et al. Aminoglycoside and glycopeptide renal toxicity in intensive care patients studied by proton magnetic resonance spectroscopy of urine. Crit Care Med 2002; 30: 1242-1245.

30 Murry KR, McKinnon PS, Mitrzyk B, Rybak MJ. Pharmacodynamic characterization of nephrotoxicity associated with once-daily aminoglycoside. Pharmacotherapy 1999; 19: 1252-1260.

31 Montero A, Ariza J, Corbella X, et al. Efficacy of colistin versus $\beta$-lactams, aminoglycosides, and rifampin as monotherapy in a mouse model of pneumonia caused by multiresistant Acinetobacter baumannii. Antimicrob Agents Chemother 2002; 46: 1946-1952.

32 Kasiakou SK, Michalopoulos A, Soteriades ES, Samonis G, Sermaides GJ, Falagas ME. Combination therapy with intravenous colistin for management of infections due to multidrug-resistant Gram-negative bacteria in patients without cystic fibrosis. Antimicrob Agents Chemother 2005; 49: 3136-3146.

33 Linden PK, Kusne S, Coley K, Fontes P, Kramer DJ, Paterson D. Use of parenteral colistin for the treatment of serious infection due to antimicrobial-resistant Pseudomonas aeruginosa. Clin Infect Dis 2003; 37: 154-160.

34 Chastre J, Fagon JY. Ventilator-associated pneumonia. Am J Respir Crit Care Med 2002; 165: 867-903. 